\title{
Analytic solution for size-dependent behaviors of micro-beam under forced vibration
}

\author{
Shuai WANG, Zhiyong WANG, Feifei WANG, Bo ZHOU*, Shifeng XUE \\ College of Pipeline and Civil Engineering, China University of Petroleum (East China), Qingdao 266580, P.R. China
}

*Corresponding Author: Bo ZHOU, Email: zhoubo@upc.edu.cn

\begin{abstract}
:
This paper focuses on the size-dependently mechanical behaviors of a micro-beam under forced vibration. Governing equations of a micro-beam under forced vibration are established by using the modified couple stress theory, Bernoulli-Euler beam theory and D'Alembert's principle together. A simply supported micro-beam under forced vibration is solved according to the established governing equations and the method of separation of variables. The dimensionless deflection, amplitude mode and period mode are defined to investigate the size-dependently mechanical behaviors of a micro-beam under forced vibration. Results show that the performance of a micro-beams under forced vibration is distinctly size-dependent when the ratio of micro-beam height to material length-scale parameter is small enough. Both frequency ratio and loading location are the important factors that determine the sizedependent performance of a micro-beams under forced vibration.
\end{abstract}

Keywords: micro-beam; forced vibration; size effect, modified couple stress theory; Bernoulli-Euler beam theory.

\section{Introduction}

Microbeams have been widely employed in micro-electromechanical systems (MEMS) ${ }^{[1-3]}$. There have been many applications of microbeams, such as microscale sensors ${ }^{[4-6]}$, microscale actuators ${ }^{[7-8]}$, and microswitch ${ }^{[9]}$.

The experimental results ${ }^{[10-11]}$ have revealed the sizedependent of material behavior in micron scale. However, the classical continuum mechanics theories couldn't describe the phenomenon. Therefore strain gradient and couple stress theory have been applied to study size effect of micron devices ${ }^{[12-16]}$. In these theories, internal material length scale parameters were introduced. Ansari et al. ${ }^{[17]}$ studied the bending, buckling and free vibration responses of functionally graded Timoshenko beams based on the theory of strain gradient elasticity. They established a size-dependent beam model with five additional material length-scale parameters using Hamilton's principle. Liang et al. ${ }^{[18]}$ built a new Bernoulli-Euler beam model employing a simplified strain gradient elasticity theory and analytically solved static bending of cantilever beams, buckling and free vibration of simply supported beams. Alashti et al. ${ }^{[19]}$ derived the governing equations of Bernoulli-Euler beams by using the variational formula and Hamilton's principle, based on the couple stress model proposed by
Hadjesfandiari et al. ${ }^{[16]}$, and discussed the problems of static bending and free vibration of Bernoulli-Euler beams with different boundary conditions.

Yang et al. ${ }^{[20]}$ presented a modified couple stress theory (MCST) based on couple stress theory. This theory revealed that couple stress tensor was symmetric and conjugated with symmetric curvature tensor to total strain energy of the system. In addition, only one material lengthscale parameter was used in the MCST, thus this theory was utilized more widely. Park et al. ${ }^{[21]}$ provided a variational formulation for the MCST using the principle of minimum total potential energy. Park et al. ${ }^{[22]}$ studied bending of Bernoulli-Euler microbeam on the basis of the MCST. The governing equation and boundary condition were developed using the principle of minimum total potential energy. Ma et al. ${ }^{[23]}$ developed a micro Timoshenko model using Hamilton's principle and variational formulation based on the MCST. Kong et al. ${ }^{[24]}$ obtained the dynamic equilibrium governing equation, initial condition and boundary condition of Bernoulli-Euler micro-beam combining the basic equation of MCST with Hamilton principle. The boundary value problem of simply supported beam and cantilever beam were solved, and size effect on natural frequencies of beams was studied. MohammadAbadi et al. ${ }^{[25]}$ analyzed free vibration of composite 
laminated beams using the modified couple stress theory. The governing equation and boundary condition were obtained by applying Hamilton's principle. The differences between Bernoulli -Euler, Timoshenko and Reddy beam models were studied. Dehrouyeh-Semnani et al. ${ }^{[26]}$ obtained Bernoulli-Euler beam model and Timoshenko beam model based on modified couple stress. The effects of size-dependent shear deformation on static bending, buckling and free vibration characteristics of micro-beams were studied.

In summary, many works have been published to investigate the effects of size-dependent on static bending, bulking and free vibration of micro-beams. However the works about the size-dependent behaviors of a micro-beam under forced vibration are few. The objective of this paper is to investigate the size-dependent behaviors of a microbeam under forced vibration. In Section 2, the modified couple stress theory is briefly introduced for considering the size effect of a micro-beam. In Section 3, the governing equations for a micro-beam under forced vibration are established by using the MCST and D'Alembert's principle together. In Section 4, a simply supported micro-beam under forced vibration is solved based on the method of separation of variables. In Section 5, the size-dependent mechanical behaviors of a micro-beam under forced vibration are numerically investigated. Finally, several important conclusions related to the size-dependent mechanical behaviors of a micro-beam under forced vibration are summarized in Section 6.

\section{Modified couple stress theory}

According to the MCST, the constitutive equation of an elastomer reads as

$\sigma_{i j}=\lambda \varepsilon_{k k} \delta_{i j}+2 G \varepsilon_{i j}$

$m_{i j}=2 l^{2} G \chi_{i j}$

where 1 is the length-scale parameter; $\sigma_{\mathrm{ij}}$ and $\varepsilon_{\mathrm{ij}}$ are stress tensor and strain tensor, respectively; $\mathrm{m}_{\mathrm{ij}}$ and $\chi_{\mathrm{ij}}$ are deviatoric part of couple stress tensor and symmetric curvature tensor, respectively; $\lambda$ and $G$ are Lame's constants expressed as

$$
\begin{aligned}
& \lambda=\frac{E \mu}{(1+\mu)(1-2 \mu)} \\
& \quad \text { and } \\
& G=\frac{E}{2(1+\mu)}
\end{aligned}
$$

respectively; $E$ and $\mu$ are elastic modulus and Poisson's ratio, respectively; $l$ is the length-scale parameter, a material constant related to the size effect.

The geometric equation of an elastomer reads as

$$
\begin{aligned}
& \varepsilon_{i j}=\frac{1}{2}\left(u_{i, j}+u_{j, i}\right) \\
& \chi_{i j}=\frac{1}{2}\left(\theta_{i, j}+\theta_{j, i}\right)
\end{aligned}
$$

where $u_{i}$ and $\theta_{i}$ are the displacement tensor and rotation tensor, respectively. The rotation tensor can be expressed as $\theta_{i}=\frac{1}{2} e_{i j k} u_{k, j}$

where $e_{i j k}$ is the third order alternating tensor.

\section{Governing equations micro-beam under forced vibration}

3.1 Formulations on basic variables
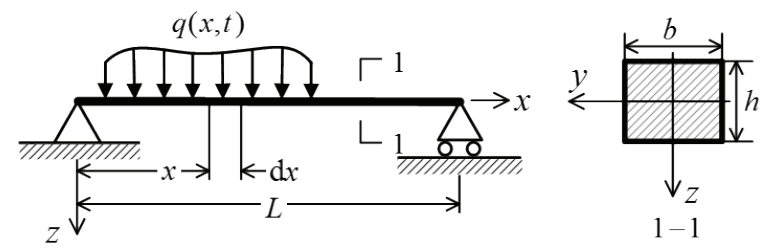

Figure 1 Bernoulli-Euler micro-beam subjected to transverse force under the Cartesian coordinate system $\mathrm{x}, \mathrm{y}, \mathrm{z}$, where $q(x, t)$ is the line density of transverse force and $t$ stands for time.

According to the theory of Bernoulli-Euler beam, the displacement field of a beam can be expressed as

$u=-z \frac{\partial w(x, t)}{\partial x}, v=0, w=w(x, t)$

where $\mathrm{u}, \mathrm{v}$ and $\mathrm{w}$ are the displacement components in directions $\mathrm{x}, \mathrm{y}$ and $\mathrm{z}$ respectively. Substituting (8) into the geometric equation (5), one formulates the nonzero strain component of a Bernoulli-Euler beam as

$\varepsilon_{x x}=-z \frac{\partial^{2} w}{\partial x^{2}}$

Substituting (8) into the rotation tensor expression (7), one has the rotation components of a Bernoulli-Euler beam

$\theta_{x}=0, \theta_{y}=-\frac{\partial w}{\partial x}, \theta_{z}=0$

Substituting (10) into the geometric equation (6), one formulates the nonzero curvature components of a Bernoulli-Euler beam as

$\chi_{x y}=\chi_{y x}=-\frac{1}{2} \frac{\partial^{2} w}{\partial x^{2}}$

Using the nonzero strain component (9) and the constitutive equation (1), one expresses the nonzero stress components of a Bernoulli-Euler beam as

$\sigma_{x x}=-(\lambda+2 G) z \frac{\partial^{2} w}{\partial x^{2}}, \sigma_{y y}=\sigma_{z z}=-\lambda z \frac{\partial^{2} w}{\partial x^{2}}$

where $\lambda$ and $G$ are Lame's constants defined by (3) and (4), respectively. Similarly using the nonzero curvature components (11) and the constitutive equation (2), one expresses the nonzero couple stress components of a Bernoulli-Euler beam as

$m_{x y}=m_{y x}=-G l^{2} \frac{\partial^{2} w}{\partial x^{2}}$

\subsection{Motion differential equation}

In order to establish the motion differential equation of the beam in Fig 1, we isolate an infinitesimal segment with a length $\mathrm{dx}$ and plots its free body diagram in Figure 2. In the 
free body diagram, $\mathrm{Q}$ and $\mathrm{M}$ are shear force and bending moment, respectively; $q$ and $f_{i}$ is the transverse force and inertial force per unit length, respectively.

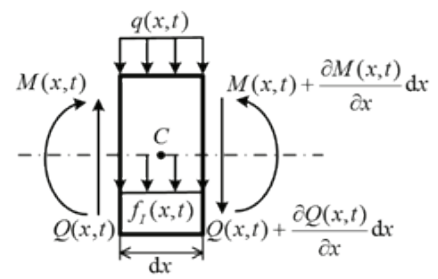

Figure 2 Free-body diagram of an infinitesimal segment with the length $\mathrm{dx}$ isolated from the micro-beam under forced vibration shown in Figure 1.

According to the D'Alembert's principle, the equilibrium of the isolated segment in Figure 2 along $\mathrm{z}$-direction requires

$Q(x, t)-q(x, t) \mathrm{d} x-\left[Q(x, t)+\frac{\partial Q(x, t)}{\partial x} \mathrm{~d} x\right]-f_{i}(x, t) \mathrm{d} x=0$

The inertial force per unit length is calculated by

$f_{i}(x, t)=-\rho A \frac{\partial^{2} w(x, t)}{\partial t^{2}}$

where $\rho$ and A are the mass density and cross-section area, respectively. Substituting (15) to (14), one has the equilibrium equation

$\frac{\partial Q(x, t)}{\partial x}-\rho A \frac{\partial^{2} w(x, t)}{\partial t^{2}}+q(x, t)=0$

The moment equilibrium equation about the central point of the isolated segment in Figure 2 reads as

$Q(x, t) \mathrm{d} x+M(x, t)-\left[M(x, t)+\frac{\partial M(x, t)}{\partial x} \mathrm{~d} x\right]=0$

The simplification on (17) relates the bending moment to shear force as

$\frac{\partial M(x, t)}{\partial x}=Q(x, t)$

Substituting (18) into (16), the equilibrium equation is rewriten as

$\frac{\partial^{2} M(x, t)}{\partial x}-\rho A \frac{\partial^{2} w(x, t)}{\partial t^{2}}+q(x, t)=0$

According to couple stress theory, the bending moment in a cross-section of the beam in Figure 1 is the result from both normal stress $\sigma_{x x}$ and couple stress $m_{x x}$ in the cross-section, namely

$M(x, t)=\int_{A} z \sigma_{x x} \mathrm{~d} A+\int_{A} m_{x y} \mathrm{~d} A$

Substituting the expression $\sigma_{x x}$ of (12) and the expression $m_{x x}$ of (13) into (20), one has

$M(x, t)=-K \frac{\partial^{2} w}{\partial x^{2}}$

where $\mathrm{K}$ is the bending stiffness of beam as

$K=(\lambda+2 G) I+G A l^{2}$

with $\mathrm{G}$ and $\lambda$ being the Lame's constants defined by (3) and (4) respectively, A being the area of cross-section and $I=\int_{A} z^{2} \mathrm{~d} A$ being the area second moment of cross-section. For a rectangular cross-section, $\mathrm{I}=\mathrm{bh}^{3} / 12$, where $\mathrm{b}$ and $\mathrm{h}$ are the width and height of the rectangular cross-section respectively. For a circular cross-section, $\mathrm{I}=\pi \mathrm{d}^{4} / 64$, where $\mathrm{d}$ is the diameter of the circular cross-section.

Substituting (21) into the equilibrium equation (19), one has the motion differential equation of a BernoulliEuler micro-beam

$\rho A \frac{\partial^{2} w(x, t)}{\partial t^{2}}+K \frac{\partial^{4} w}{\partial x^{4}}=q(x, t)$

where $\mathrm{K}$ is the bending stiffness of beam defined by (22).

\section{Solution of micro-beam under forced vibra- tion}

\subsection{Natural frequency}

Letting $\mathrm{q}(\mathrm{x}, \mathrm{t})=0$ in the motion differential equation (24), one has the free vibration differential equation of a Bernoulli-Euler beam

$\rho A \frac{\partial^{2} w(x, t)}{\partial t^{2}}+K \frac{\partial^{4} w}{\partial x^{4}}=0$

According to method of separation of variables, one can assume the solution of differential equation (25) as the form

$w(x, t)=\eta(t) \Phi(x)$

where $\Phi(\mathrm{x})$ is called as mode function, and

$\eta(t)=B \cos (\omega t)+C \sin (\omega t)$

with $\omega$ being the natural frequency, $\mathrm{B}$ and $\mathrm{C}$ being the undetermined coefficients.

Substituting (26) and (27) into (25), one has $\frac{\mathrm{d}^{4} \Phi}{\mathrm{d} x^{4}}-\beta^{4} \Phi=0$

where

$\beta^{4}=\frac{\rho A \omega^{2}}{K}$

The general solution of the mode differential equation (28) reads as

$\Phi(x)=C_{1} \sin (\beta x)+C_{2} \cos (\beta x)+C_{3} \sinh (\beta x)+C_{4} \cosh (\beta x)$

where $C_{1}, C_{2}, C_{3}$ and $C_{4}$ are the undetermined coefficients.

For the simply supported beam in Figure 1, the displacement boundary conditions are

$\left.\Phi\right|_{x=0}=\left.\Phi\right|_{x=L}=0,\left.\quad \frac{\mathrm{d}^{2} \Phi}{\mathrm{d} x^{2}}\right|_{x=0}=\left.\frac{\mathrm{d}^{2} \Phi}{\mathrm{d} x^{2}}\right|_{x=L}=0$

Substituting the mode equation (30) into the displacement boundary conditions (31) leads to $\mathrm{C}_{2}=\mathrm{C}_{3}=$ $\mathrm{C}_{4}=0$, and

$\sin (\beta L)=0$

$\beta=\frac{i \pi}{L} \quad(i=1,2,3, \cdots)$

Then one can express the mode functions of the simply supported beam as 
$\Phi_{i}(x)=\sin \left(\frac{i \pi x}{L}\right) \quad(i=1,2,3, \cdots)$

when $C_{1}=1$. From (29) and (33), one has the natural frequency of the simply supported beam

$\omega_{i}=(i \pi)^{2} \sqrt{\frac{K}{\rho A L^{4}}} \quad(i=1,2,3, \cdots)$

where $\mathrm{K}$ is the bending stiffness of beam defined by (22).

\subsection{Orthogonality of mode functions}

Using (28) and (29), one has

$\frac{\mathrm{d}^{2} R_{i}}{\mathrm{~d} x^{2}}=\omega_{i}^{2} \rho A \Phi_{\mathrm{i}}(x)$

where $\Phi_{\mathrm{i}}$ and $\omega_{\mathrm{i}}$ are the $\mathrm{i}^{\text {th }}$ order mode and natural frequency of the simply supported Bernoulli-Euler microbeam in Figure 1 respectively, and

$R_{i}(x)=K \frac{\mathrm{d}^{2} \Phi_{i}}{\mathrm{~d} x^{2}}$

with $\mathrm{K}$ being the bending stiffness after (22).

Multiplying both sides of (36) by the $\mathrm{j}^{\text {th }}$ order mode function $\Phi_{j}(\mathrm{x})$ and integrating them along the beam length, one has

$\int_{0}^{L} \Phi_{\mathrm{j}}(x) \frac{\mathrm{d}^{2} R_{i}}{\mathrm{~d} x^{2}} d x=\int_{0}^{L} \Phi_{j}(x) \omega_{i}^{2} \rho A \Phi_{\mathrm{i}}(x) \mathrm{d} x$

For the left side of (38),

$\int_{0}^{L} \Phi_{j}(x) \frac{\mathrm{d}^{2} R_{i}}{\mathrm{~d} x^{2}} \mathrm{~d} x=\left.\Phi_{j}(x) \frac{\mathrm{d} R_{i}}{\mathrm{~d} x}\right|_{0} ^{L}-\left.\frac{\mathrm{d} \Phi_{j}}{\mathrm{~d} x} R_{i}(x)\right|_{0} ^{L}+\int_{0}^{L} \frac{\mathrm{d}^{2} \Phi_{j}}{\mathrm{~d} x^{2}} R_{i}(x) \mathrm{d} x$

The boundary conditions of the simply supported beam in Figure 1 gives

$\left.\Phi_{j}(x) R^{\prime}(x)\right|_{0} ^{L}=\left.\Phi_{j}^{\prime}(x) R(x)\right|_{0} ^{L}=0$

Substituting (40) and (37) into (39), one has

$\int_{0}^{L} \Phi_{j}(x) \frac{\mathrm{d}^{2} R_{i}}{\mathrm{~d} x^{2}} \mathrm{~d} x=\int_{0}^{L} K \frac{\mathrm{d}^{2} \Phi_{j}}{\mathrm{~d} x^{2}} \frac{\mathrm{d}^{2} \Phi_{i}}{\mathrm{~d} x^{2}} \mathrm{~d} x$

where $\mathrm{K}$ being the bending stiffness after (22).

From (38) and (41), one has

$K \int_{0}^{L} \frac{\mathrm{d}^{2} \Phi_{j}}{\mathrm{~d} x^{2}} \frac{\mathrm{d}^{2} \Phi_{i}}{\mathrm{~d} x^{2}} \mathrm{~d} x=\omega_{i}^{2} \rho A \int_{0}^{L} \Phi_{j}(x) \Phi_{\mathrm{i}}(x) \mathrm{d} x$

Interchanging the subscripts $i$ and $j$ in (42) leads to

$K \int_{0}^{L} \frac{\mathrm{d}^{2} \Phi_{i}}{\mathrm{~d} x^{2}} \frac{\mathrm{d}^{2} \Phi_{j}}{\mathrm{~d} x^{2}} \mathrm{~d} x=\omega_{j}^{2} \rho A \int_{0}^{L} \Phi_{i}(x) \Phi_{\mathrm{j}}(x) \mathrm{d} x$

From (42) and (43), one has

$\left(\omega_{i}^{2}-\omega_{j}^{2}\right) \int_{0}^{L} \Phi_{j}(x) \Phi_{\mathrm{i}}(x) \mathrm{d} x=0$

The standard mode function of $\mathrm{i}^{\text {th }}$ order is defined as $\varphi_{i}(x)=\frac{\Phi_{i}(x)}{\sqrt{m_{i}}}$

where

$m_{i}=\rho A \int_{0}^{L} \Phi_{i}^{2}(x) \mathrm{d} x$

Using (45), (46) and (44), one has

$\int_{0}^{L} \rho A \varphi_{i}(x) \varphi_{j}(x) \mathrm{d} x=\delta_{i j}$, where $\delta_{\mathrm{ij}}$ is the Kronecker delta symbol . (47) illustrates the orthogonality of mode functions.

\subsection{Solution of forced vibration}

According to the method of mode superposition, one can set the solution of the motion differential equation (24) as

$w(x, t)=\sum_{j=1}^{\infty} \eta_{j}(t) \varphi_{j}(x)$

where $\varphi_{j}(\mathrm{x})$ is the $\mathrm{j}^{\text {th }}$ order standard mode function expressed by (45). Substituting (48) into the motion differential equation (24), one has

$\rho A \sum_{j=1}^{\infty} \varphi_{j}(x) \frac{\mathrm{d}^{2} \eta_{j}}{\mathrm{~d} t^{2}}+K \sum_{j=1}^{\infty} \frac{\mathrm{d}^{4} \varphi_{j}}{\mathrm{~d} x^{4}} \eta_{j}(t)=q(x, t)$

Using (28) and (29) in (49), one has

$\rho A \sum_{j=1}^{\infty} \varphi_{j}(x) \frac{\mathrm{d}^{2} \eta_{j}}{\mathrm{~d} t^{2}}+\rho A \sum_{j=1}^{\infty} \omega_{j}^{2} \varphi_{j}(x) \eta_{j}(t)=q(x, t)$

Multiplying both sides of (50) by the $\mathrm{i}^{\text {th }}$ order standard mode function $\varphi_{i}(\mathrm{x})$ and integrating them along the beam length with using the orthogonality of standard mode functions expressed in (47), one has

$\frac{\mathrm{d}^{2} \eta_{i}}{\mathrm{~d} t^{2}}+\omega_{i}^{2} \eta_{i}(t)=Q_{i}(t)$

where

$Q_{i}(t)=\int_{0}^{L} q(x, t) \varphi_{i}(x) \mathrm{d} x$

According to (48), the initial condition of the simply supported micro-beam in Figure 1 can be expressed as $\left.w(x, t)\right|_{t=0}=\left.\left.\sum_{j=1}^{\infty} \eta_{j}(t)\right|_{t=0} \varphi_{j}(x) \quad \frac{\partial w(x, t)}{\partial t}\right|_{t=0}=\left.\sum_{j=1}^{\infty} \frac{\mathrm{d} \eta_{j}}{\mathrm{~d} t}\right|_{t=0} \varphi_{j}(x)$

Multiplying both sides of (53) by the $\mathrm{i}^{\text {th }}$ order standard mode function $\varphi_{\mathrm{i}}(\mathrm{x})$ and integrating them along the beam length with using the orthogonality of standard mode functions in (47), one has

$\left.\eta_{i}(t)\right|_{t=0}=\left.\left.\int_{0}^{L} w(x, t)\right|_{t=0} \varphi_{i}(x) \mathrm{d} x \frac{\mathrm{d} \eta}{\mathrm{d} t}\right|_{t=0}=\left.\int_{0}^{L} \frac{\partial w}{\partial t}\right|_{t=0} \varphi_{i}(x) \mathrm{d} x$

Using the Duhamel integral, one obtains the solution of the differential equation (51) as

$\eta_{i}(t)=\frac{1}{\omega_{i}} \int_{0}^{t}\left[Q_{i}(\tau) \sin \omega_{i}(t-\tau)\right] \mathrm{d} \tau+\left.\eta_{i}(t)\right|_{t=0} \cos \omega_{i} t+\left.\frac{\mathrm{d} \eta}{\mathrm{d} t}\right|_{t=0} \frac{\sin \omega_{i} t}{\omega_{i}}$

Substituting (55) and (45) into (48) gives the solution of the Bernoulli-Euler simply supported micro-beam under forced vibration.

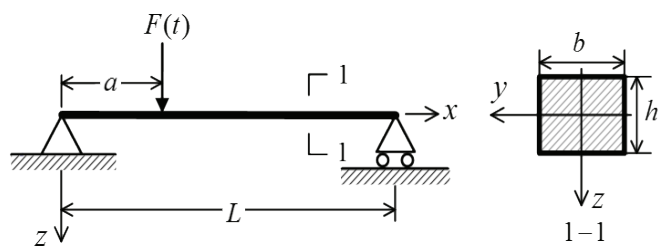

Figure 3 A simply supported Bernoulli-Euler micro-beam with rectangular section subjected a concentrated force, where $\mathrm{F}(\mathrm{t})$ is the concentrated force functioned by (56) and $\mathrm{a}$ is the distance between the point concentrated force applied and the left-hand side of beam. 
Figure 3 shows a simply supported Bernoulli-Euler micro-beam subjected to a concentrated force formulated as

$$
F(t)=P \sin \left(\omega_{p} t\right)
$$

then the line density of distributed load is formulated as

$q(x, t)= \begin{cases}0 & \text { others } \\ \frac{P(t)}{\Delta} & (a \leq x \leq a+\Delta)\end{cases}$

where $\Delta$ is an infinitesimal value. Substituting (57) into (52), one has

$Q_{i}(t)=P \sqrt{\frac{2}{\rho A L}} \sin \left(\omega_{p} t\right) \sin \left(\frac{i \pi a}{L}\right)$

Substituting (58) into (55) and using the initial conditions

$$
\begin{aligned}
& \left.\eta_{i}(t)\right|_{t=0}=0 \text { and }\left.\frac{\mathrm{d} \eta}{\mathrm{d} t}\right|_{t=0}=0 \\
& \text { one has } \\
& \eta_{i}=\frac{P}{\omega_{i}^{2}-\omega_{p}^{2}} \sqrt{\frac{2}{\rho A L}} \sin \left(\frac{i \pi a}{L}\right)\left[\sin \left(\omega_{p} t\right)-\frac{\omega_{p}}{\omega_{i}} \sin \left(\omega_{i} t\right)\right]
\end{aligned}
$$

Substituting (60) and (45) into (48) and using (34), one has the solution of forced vibration of the micro-beam in Figure 3,

$$
w(x, t)=\sum_{i=1}^{\infty} \frac{2 P}{\rho A L\left(\omega_{i}^{2}-\omega_{p}^{2}\right)} \sin \left(\frac{i \pi x}{L}\right) \sin \left(\frac{i \pi a}{L}\right)\left[\sin \left(\omega_{p} t\right)-\frac{\omega_{p}}{\omega_{i}} \sin \left(\omega_{i} t\right)\right]
$$

In order to investigate the size effect of the forced vibration of micro-beam conveniently, the dimensionless deflection is defined as

$$
w^{\prime}(x, t)=w(x, t) /\left(\frac{2 P}{\rho g A}\right)
$$

Substituting (61) into (62), the dimensionless deflection can be expressed as $w^{\prime}(x, t)=\sum_{i=1}^{\infty} W_{i}(x) \Psi_{i}(t)$

where

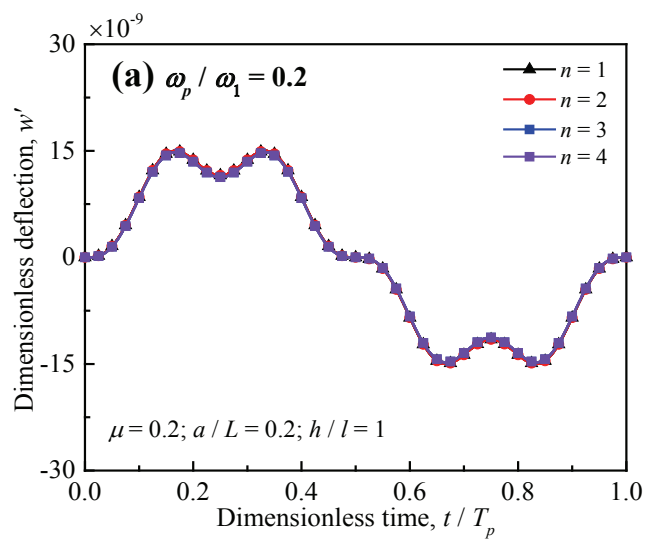

$W_{i}(x)=\frac{g}{L\left(\omega_{i}^{2}-\omega_{p}^{2}\right)} \sin \left(\frac{i \pi a}{L}\right) \sin \left(\frac{i \pi x}{L}\right)$

is called the $\mathrm{i}^{\text {th }}$ order dimensionless amplitude mode and

$\Psi_{i}(t)=\sin \left(\omega_{p} t\right)-\frac{\omega_{p}}{\omega_{i}} \sin \left(\omega_{i} t\right)$

is called the $\mathrm{i}^{\text {th }}$ order dimensionless period mode.

\section{Analysis on forced vibration of micro-beam}

In this section the micro-beam under forced vibration in Figure 3 is numerically investigated to reveal the size effects and the influences of Poisson's ratio, frequency ratio and loading location. The frequency ratio is defined as $\omega_{\mathrm{p}} / \omega_{1}$, where $\omega_{p}$ and $\omega_{1}$ are the load frequency and the first order natural frequency of the micro-beam, respectively.

Figure 4 shows the curves of dimensionless deflection versus dimensionless time of a point in the micro-beam under forced vibration in Figure 3 based on the front 1, 2, 3 and 4 orders of dimensionless modes of amplitude and period, respectively. The dimensionless deflection is calculated by (63) with $\mathrm{x}=\mathrm{L} / 2$, and the dimensionless modes of amplitude and period are determined by (64) and (65), respectively. Figure 4(a) plots the curves during the period $T_{p}=2 \pi / \omega_{p}$ when the value of frequency ratio is 0.2 . It is found that the curves based on the front 1, 2, 3 and 4 orders of dimensionless amplitude and period modes are very close and similar. Figure 4(b) plots the curves during the period $T_{1}=2 \pi / \omega_{1}$ when the value of frequency ratio is 2.0 , we can also see that the dimensionless amplitudes and periodic modes of the front 1, 2, 3, and 4 orders are very close. This means the first order dimensionless modes of amplitude and period are the main contribution to the solution of the micro-beam under forced vibration. Therefore the forced vibration of the micro-beam are calculated using only the first order dimensionless modes of amplitude and period in the following sections.

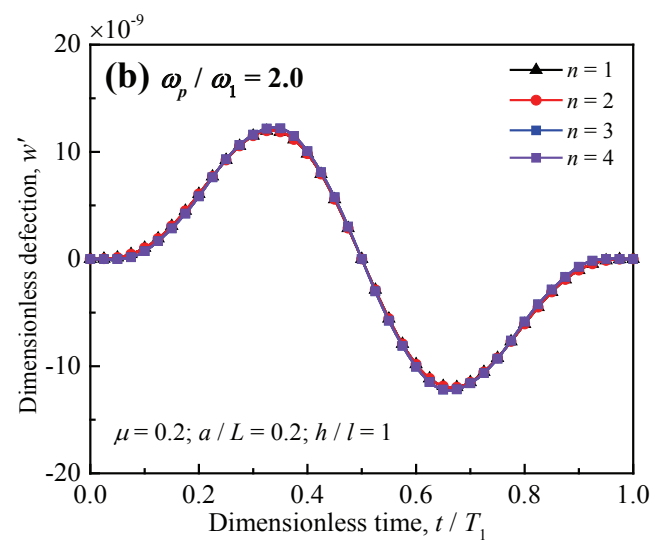

Figure 4 Curves of dimensionless deflection vs. dimensionless time of a point according to the front 1, 2, 3 and 4 orders of dimensionless amplitude and period modes, respectively.

On the other hand, comparisons between Figure 4(a) and Figure 4(b) illustrate that the frequency ratio is an important factor determining the performance of the micro-beam under forced vibration. Although when the frequency ratio is changed, the curve as a whole shows an "increasing-decreasing-increasing" trend, a small 
frequency ratio will cause more fluctuations, and changing the frequency ratio can change the variation range of the dimensionless deflection.

\subsection{Influences of frequency ratio}

Figure 5 shows the curves of first order dimensionless amplitude mode of the micro-beam under forced vibration in Figure 3 with respect to the different values of frequency ratio. Figure 5(a) plots the curves when the value of frequency ratio is below 1.0. It is clear that each curve forms a hump and the altitude of hump rises with the increased value of frequency ratio when the value of frequency ratio is below 1.0. This means that the flexibility of the microbeam under forced vibration increases with the increased value of frequency ratio when the value of frequency ratio is below 1.0. Figure 5(b) plots the curves when the value of frequency ratio is above 1.0. It is clear that each curve forms a hump and the altitude of hump declines with the increased value of frequency ratio when the value of frequency ratio is above 1.0. This means that the flexibility of the micro-beam under forced vibration decreases with the increased value of frequency ratio when the value of frequency ratio is above 1.0.

Figure 6 shows the curves of first order dimensionless period of the micro-beam under forced vibration in Figure 3 with respect to the different values of frequency ratio. Figure 6(a) plots the curves when the value of frequency ratio is below 1.0. Figure 6(b) plots the curves when the value of frequency ratio is above 1.0. It is found that the frequency ratio has significant influence on the period mode of micro-beam under forced vibration.

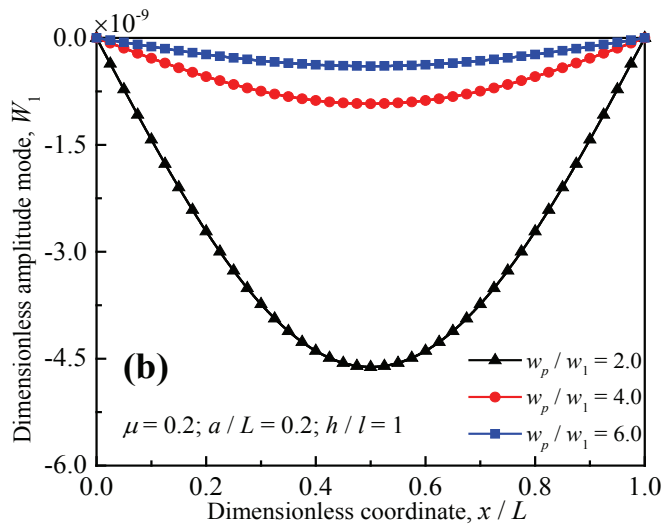

Figure 5 Curves of first order dimensionless amplitude mode of the micro-beam under forced vibration with respect to the different values of frequency ratio.
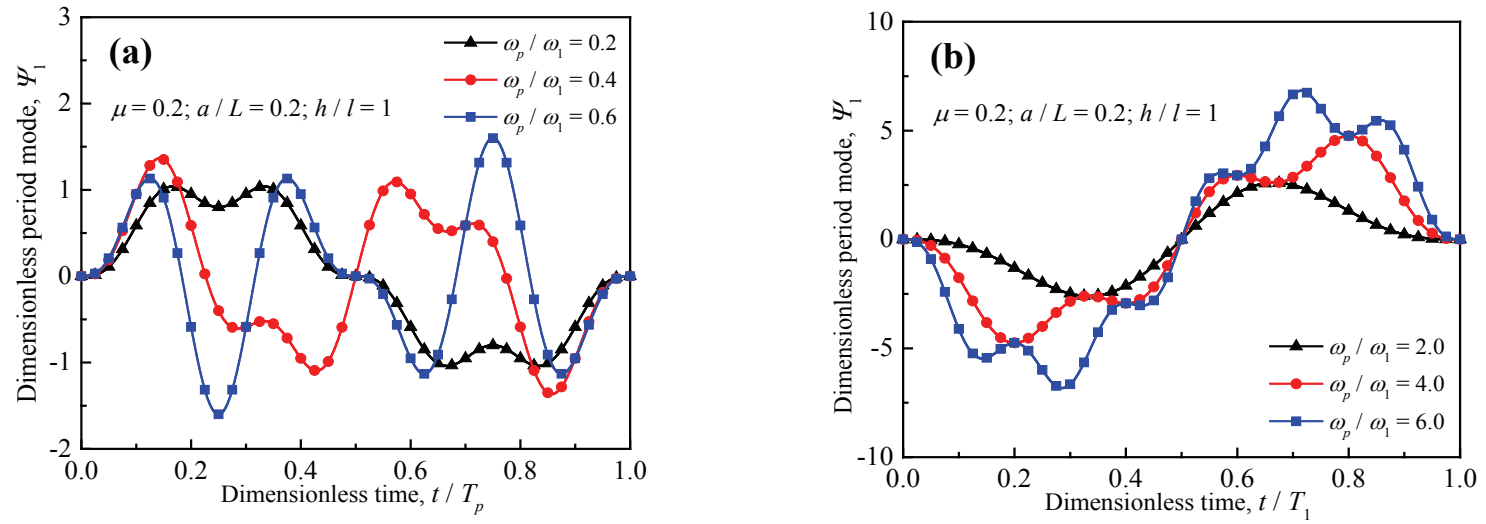

Figure 6 Curves of first order dimensionless period mode of the micro-beam under forced vibration with respect to the different values of frequency ratio.

Figure 7 shows that the curves of dimensionless deflection versus dimensionless time of a point in the micro-beam under forced vibration in Figure 3 with respect to the different values of frequency ratio. Figure 7 (a) plots the curves when the value of frequency ratio is below 1.0. Figure 7 (b) plots the curves when the value of frequency ratio is above 1.0. It is clear that the frequency ratio has a significant influence on the performance of the microbeam under forced vibration.

\subsection{Size effect of forced vibration}

Figure 8 shows the curves of the first order dimensionless amplitude mode of the micro-beam under forced vibration in Figure 3 with respect to the different values of dimensionless height, where $\mathrm{L}$ and $\mathrm{h}$ are the length and height of the micro-beam, respectively, and 1 is the material length-scale parameter. The first order dimensionless amplitude mode is calculated by (64) and the dimensionless 
height is defined as the ratio of the height of micro-beam to the material length-scale parameter h/l. Figure 8(a) plots the curves when the value of frequency ratio is 0.2 . It is clear that each curve is with a shape of hump, and the altitude of hump increases with the increased value of dimensionless height. This illustrates that the flexibility of the micro-beam

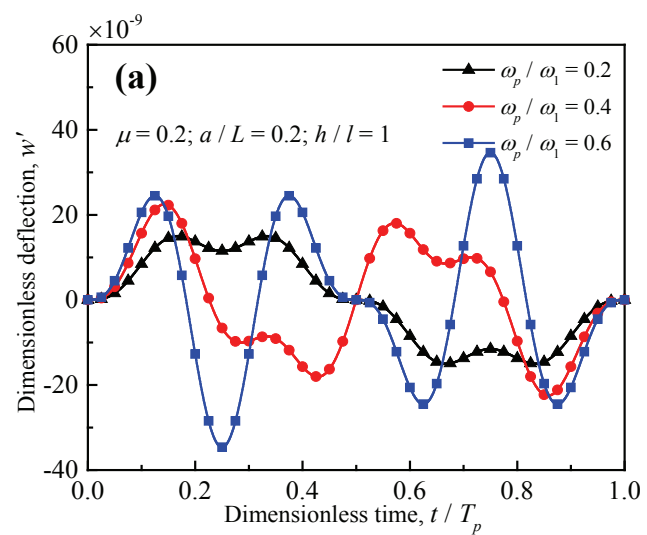

under forced vibration rises with the increased value of dimensionless height. Figure 8(b) plots the curves when the value of frequency ratio is 2.0. The comparisons between Figure 8(a) and Figure 8(b) show that the frequency ratio has a significant influence on the amplitude mode of the micro-beam under forced vibration.

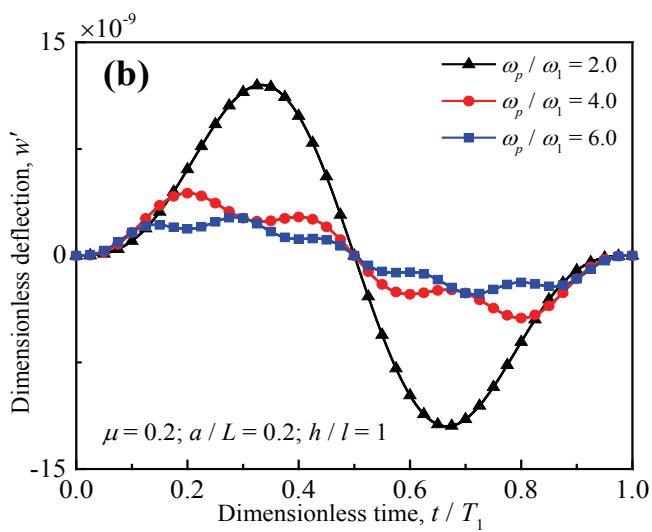

Figure 7 Curves of dimensionless deflection vs. dimensionless time of a point in the micro-beam with respective to the different values of frequency ratio.
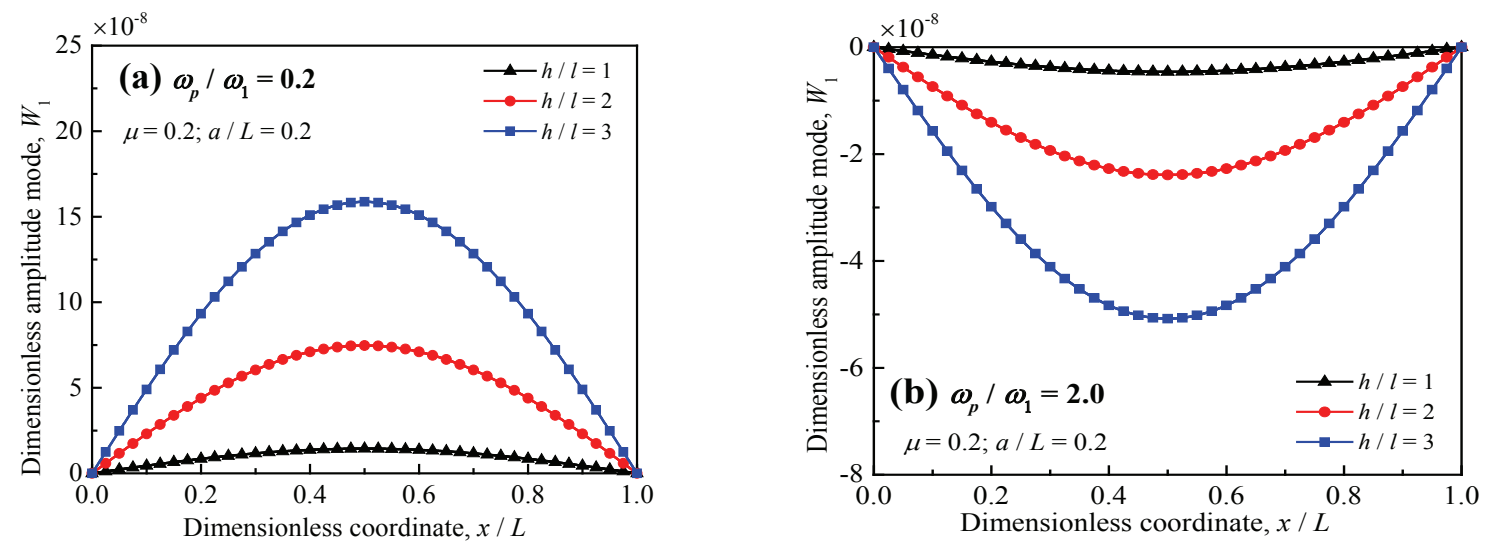

Figure 8 Curves of first order dimensionless amplitude mode of the micro-beam under forced vibration with respect to the different values of dimensionless height.
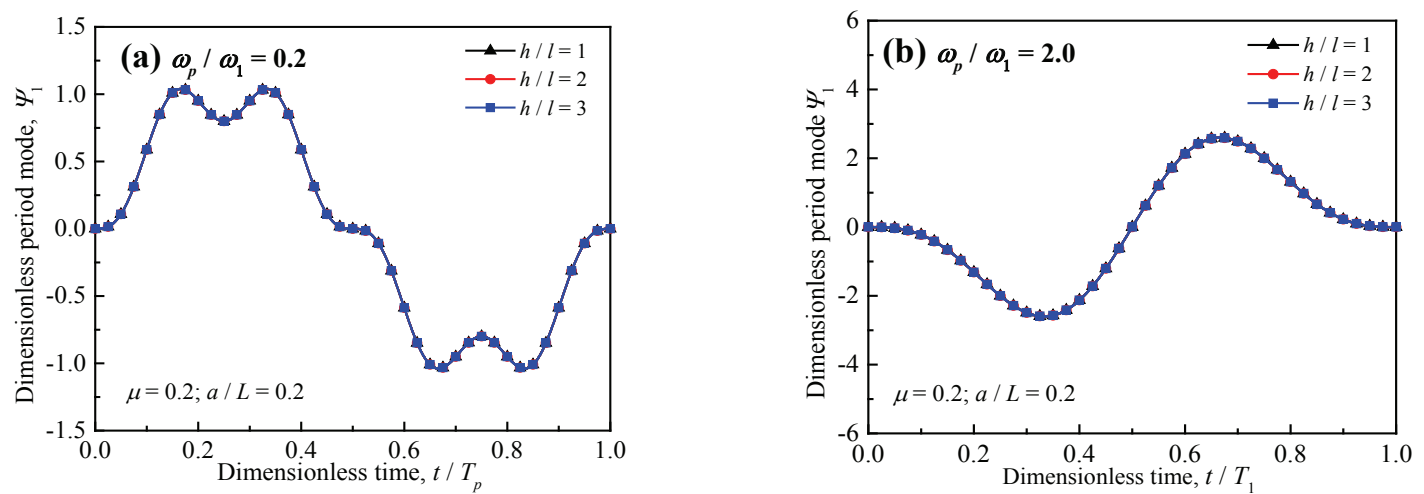

Figure 9 Curves of first order dimensionless period mode of the micro-beam under forced vibration with respect to the different values of dimensionless height.

Figure 9 shows the curves of first order dimensionless period mode of the micro-beam under forced vibration in Figure 3 with respect to the different values of dimensionless height, where the first order dimensionless period mode is calculated by (65). Figure 9(a) plots the curves during the period $T_{p}=2 \pi / \omega_{p}$ when the value of frequency ratio is 0.2 . 
It is clear that the curves with respect to the different values of dimensionless height are very close and similar, which illustrates that the dimensionless height has little influence on the period mode of the micro-beam under forced vibration. Figure 9 (b) plots the curves during the period $\mathrm{T}_{1}=2 \pi / \omega_{1}$ when the value of frequency ratio is 2.0 . The comparisons between Figure 9(a) and Figure 9(b) show that the frequency ratio $\omega_{\mathrm{p}} / \omega_{1}$ has a significant influence on the period mode of the micro-beam under forced vibration.

Figure 10 shows the curves of dimensionless deflection versus dimensionless time of a point in the microbeam under forced vibration in Figure 3 with respect to the different values of dimensionless height, where the dimensionless deflection is calculated by (63) with $x=L / 2$ based on the first order modes of dimensionless amplitude and period. Figure 10(a) plots the curves during the period $T_{p}=2 \pi / \omega_{p}$ when the value of frequency ratio is 0.2 . It is clear that the curve vertically extends with the increased value of dimensionless height. This is due to that the flexibility

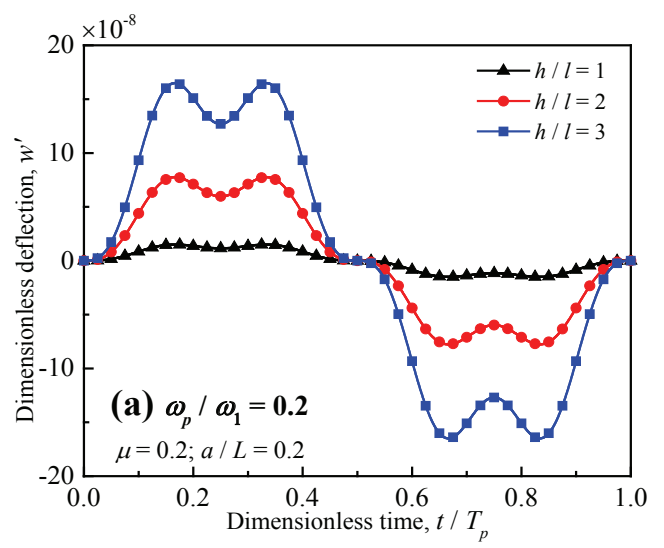

of the micro-beam increases with the increased value of dimensionless height. Figure 10(b) plots the curves during the period $T_{1}=2 \pi / \omega_{1}$ when the value of frequency ratio is 2.0. The comparisons between Figure 10(a) and Figure $10(\mathrm{~b})$ show that the frequency ratio $\omega_{\mathrm{p}} / \omega_{1}$ has a significant influence on the performance of the micro-beam under forced vibration.

\subsection{Influences of loading location}

Figure 11 shows that the curves of first order dimensionless amplitude mode of the micro-beam under forced vibration in Figure 3 with respect to the different loading locations. Figure 11(a) and Figure 11(b) plot the curves when the values of frequency are 0.2 and 2.0, respectively. It is found that the loading location has an obvious influence on the amplitude mode of the micro-beam under the forced vibration. It is also found that the frequency ratio has an obvious influence on the dimensionless amplitude mode of the micro-beam under the forced vibration.

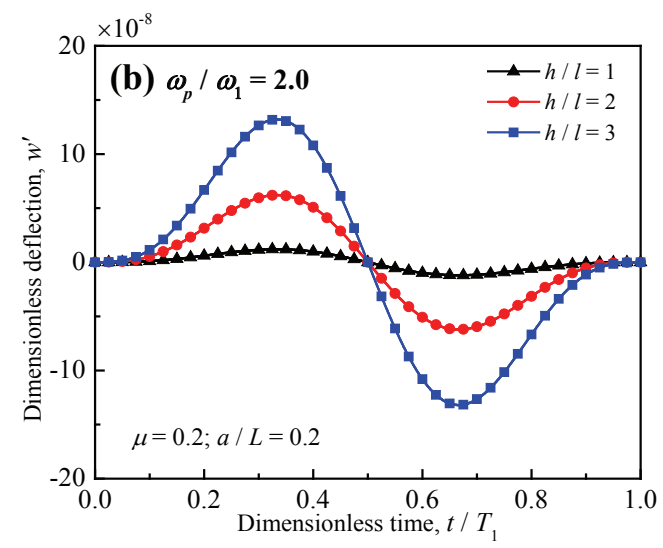

Figure 10 Curves of dimensionless deflection vs. dimensionless time of a point in the micro-beam with respect to the different values of dimensionless height.
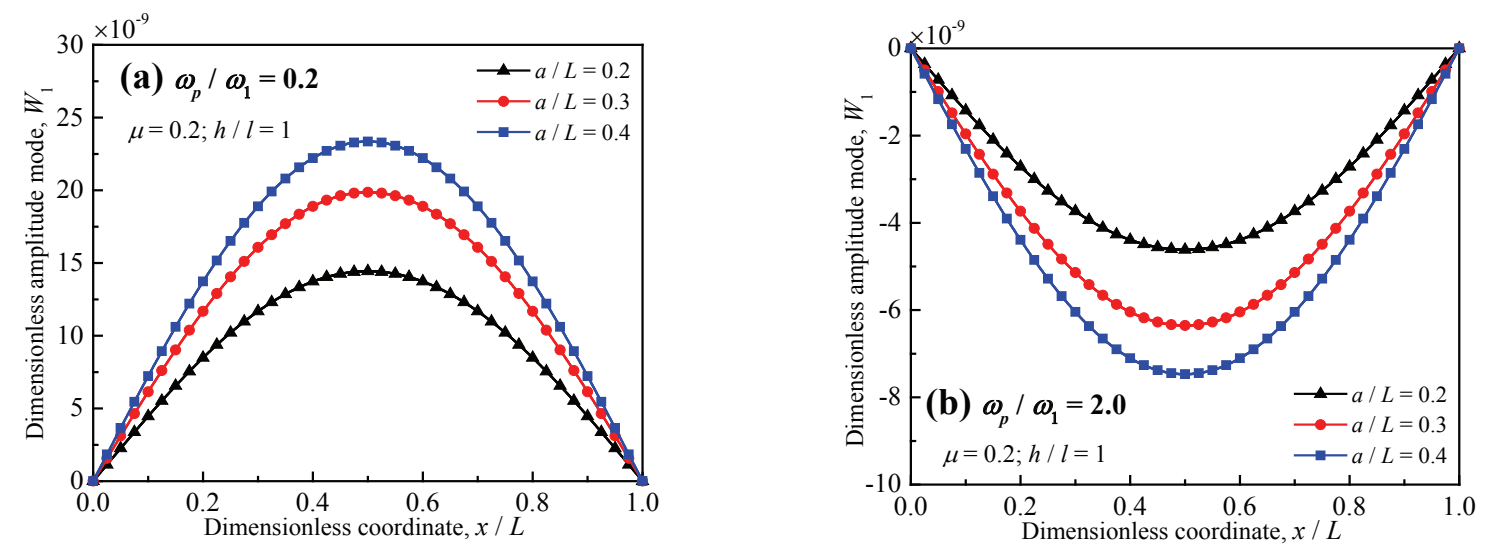

Figure 11 Curves of first order dimensionless amplitude mode of the micro-beam under forced vibration with respect to the different location load applied.

Figure 12 shows the curves of first order dimensionless period mode of the micro-beam under forced vibration in Figure 3 with respect to the different loading locations. Figure 12(a) and Figure 12(b) plot the curves when the values of frequency are 0.2 and 2.0, respectively. It is found that the loading location has little influence on the period mode of the micro-beam under the forced vibration. It is also clear that the frequency ratio has a significant influence on the period mode of the micro-beam under the forced vibration.

Figure 13 shows the curves of dimensionless deflection 
versus dimensionless time of the micro-beam under forced vibration in Figure 3 with respect to the different loading locations. Figure 13(a) and Figure 13(b) plot the curves when the values of frequency are 0.2 and 2.0, respectively. It is found that the loading location has obvious influence

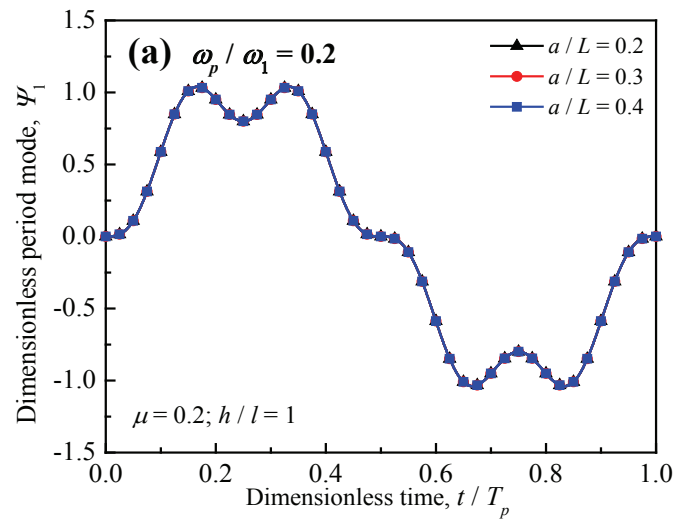

on the performance of the micro-beam under the forced vibration. It is also clear that the frequency ratio has obvious influence on the performance of the micro-beam under the forced vibration.

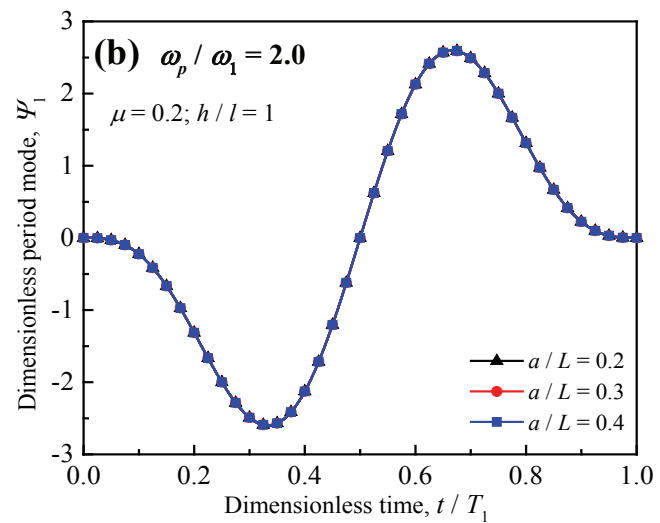

Figure 12 Curves of first order dimensionless period mode of the micro-beam under forced vibration with respect to the different location load applied.
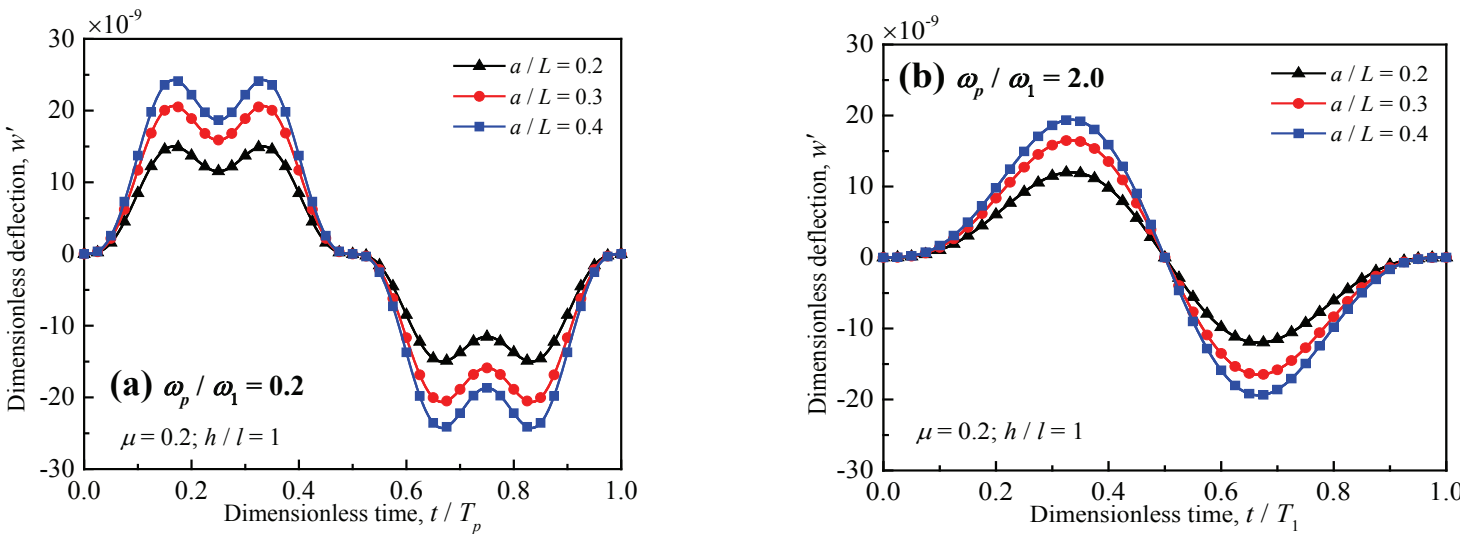

Figure 13 Curves of dimensionless deflection vs. dimensionless time of a point in the micro-beam with respect to the different location load applied.

\section{Conclusions}

The components of strain, curvature, stress and couple stress of a micro-beam are formulated according to the MCST and Bernoulli-Euler beam theory. The motion differential equation of a micro-beam is derived by using the D'Alembert's principle. The solution of a micro-beam under forced vibration is obtained by using the method of separation of variables. Based on the numerical results, the important conclusions related to the behaviors of a microbeam under forced vibration are summarized as follows.

1) The size effects of a micro-beam under forced vibration, which includes the size effects of deflection, amplitude mode and period mode, are obvious when the value of dimensionless height is small enough.

2) The frequency ratio has a significant influence on both dimensionless modes of amplitude and period of a micro-beam under forced vibration, therefore it is the important factor that determines the behaviors of a micro- beam under forced vibration.

3) The loading location has a significant influence on the dimensionless amplitude mode of a micro-beam under the forced vibration, however its influence on the dimensionless period mode of a micro-beam under the forced vibration is negligible.

Author Contributions: Bo ZHOU first proposed a method to analyze the size-dependently mechanical behaviors of a micro-beam under forced vibration by using modified couple stress theory, Bernoulli-Euler beam theory and D'Alembert's principle. Shuai WANG solved a simply supported micro-beam under forced vibration according to the established governing equations and the method of separation of variables, and defined the dimensionless deflection, amplitude mode and period mode to investigate the size-dependently mechanical behaviors of a microbeam under forced vibration. Zhiyong WANG established the governing equations of a micro-beam under forced 
vibration. Feifei WANG summarized the results of numerical simulation. Shifeng XUE put forward some guiding opinions on the logicality and innovation of the article.

Conflict of Interest: We declare that there is no conflict of interest regarding the publication of this paper.

Acknowledgments: The authors of this paper acknowledge the supports from the National Key Research and Development Program of China (Grant No. 2017YFC0307604) and the Talent Foundation of China University of Petroleum (Grant No. Y1215042).

This is the Appendix: This article does not cover the details that require an appendix.

\section{References}

[1] Chen, S., Baughn, T. V., Yao, Z. J., and Goldsmith, C. L. A new in situ residual stress mea-surement method for a mems thin fixed-fixed beam structure. Journal of Microelectromechanical Systems, 11(4), 309-316 (2002)

[2] Li, M., Huang, Q. A., and Li, W. H. A nodal analysis method for electromechanical behavior simulation of bow-tie shaped microbeams. Microsystem Technologies, 15(7), 985-991 (2009)

[3] Dai, H. L., Wang, Y. K., and Wang, L. Nonlinear dynamics of cantilevered microbeams based on modified couple stress theory. International Journal of Engineering Science, 94(2), 103-112 (2015)

[4] Muniraj, N. J. R. Mems based humidity sensor using Si cantilever beam for harsh environmental conditions. Microsystem Technologies, 17(1), 27-29 (2011)

[5] Nallathambi, A., and Shanmuganantham, T. Performance analysis of cantilever based MEMS sensor for environmental applications. 2014 International Conference on Smart Structures and Systems (ICSSS), IEEE, 2015.

[6] Shoaib, M. , Khamis, N. H. , Basheer, N. , and Tariq, M. Frequency and displacement analysis of electrostatic cantilever-based mems sensor. Analog Integrated Circuits and Signal Processing, 88(1), 1-11 (2016)

[7] Zhang, W., Meng, G., and Li, H. Adaptive vibration control of micro-cantilever beam with piezo-electric actuator in mems. International Journal of Advanced Manufacturing Technology, 28(3-4), 321-327 (2006)

[8] Li, X., Zhao, Y., Hu, T., Xu, W., Zhao, Y., and Bai, Y. Design of a large displacement thermal actuator with a cascaded v-beam amplification for mems safety-and-arming devices. Microsystem Technologies, 21(11), 2367-2374 (2015)

[9] Harsha, C. S., Prasanth, C. S., and Pratiher, B. Prediction of pull-in phenomena and structural stability analysis of an electrostatically actuated microswitch. Acta Mechanica, 227(9), 2577-2594 (2016)

[10] Fleck, N. A., Muller, G. M., Ashby, M. F., and Hutchinson, J. W. Strain gradient plasticity: theory and experiment. Acta Metal Mater, 42(2), 475-487 (1994)

[11] J.S., St"olken, and A.G., Evans. A microbend test method for measuring the plasticity length scale. Acta Materialia, 46(14), 5109-5115 (1998)

[12] Toupin, R. A. Elastic materials with couple-stresses. Archive for Rational Mechanics and Analysis, 11(1), 385414 (1962)

[13] Mindlin, R. D. Micro-structure in linear elasticity. Archive for Rational Mechanics and Analysis, 16(1), 51-78 (1964)

[14] Fleck, N. A., and Hutchinson, J. W. A reformulation of strain gradient plasticity. Journal of the Mechanics and Physics of Solids, 49(10), 2245-2271 (2001)

[15] Huang, Y., Qu, S., Hwang, K. C., Li, M., and Gao, H. A conventional theory of mechanism-based strain gradient plasticity. International Journal of Plasticity, 20(4), 753782 (2004)

[16] Hadjesfandiari, A. R., and Dargush, G. F. Couple stress theory for solids. International Journal of Solids and Structures, 48(18), 2496-2510 (2011)

[17] Ansari, R., Gholami, R., Shojaei, M. F., Mohammadi, V., and Sahmani, S. Size-dependent bend-ing, buckling and free vibration of functionally graded timoshenko microbeams based on the most general strain gradient theory. Composite Structures, 100(5), 385-397 (2013)

[18] Liang, X., Hu, S., and Shen, S. A new Bernoulli-Euler beam model based on a simplified strain gradient elasticity theory and its applications. Composite Structures, 111(1), 317-323 (2014)

[19] Alashti A. R., and Abolghasemi, A. H. A size-dependent Bernoulli-Euler beam formulation based on a new model of couple stress theory. International Journal of Engineering Transactions C, 27(6), 951-960 (2014)

[20] Yang, F., Chong, A. C. M., Lam, D. C. C., and Tong, P. Couple stress based strain gradient theory for elasticity. International Journal of Solids and Structures, 39(10), 2731-2743 (2002)

[21] Park, S. K. and Gao, X. L. Variational formulation of a modified couple stress theory and its application to a simple shear problem. Zeitschrift Fu"r Angewandte Mathematik Und Physik, 59(5), 904-917 (2008)

[22] Park, S. K. and Gao, X. L. Bernoulli-euler beam model based on a modified couple stress the-ory.Journal of Micromechanics and Microengineering, 16(11), 23552359 (2006)

[23] Ma, H. M., Gao, X. L., and Reddy, J. N. A microstructuredependent timoshenko beam model based on a modified couple stress theory. Journal of the Mechanics 
and Physics of Solids, 56(12), 3379-3391 (2008)

[24] Kong, S., Zhou, S., Nie, Z., and Wang, K. The sizedependent natural frequency of bernoulli-euler microbeams. International Journal of Engineering Science, 46(5), 427-437 (2008)

[25] Mohammad-Abadi, M., and Daneshmehr, A. R. Modified couple stress theory applied to dynamic analysis of composite laminated beams by considering different beam theories. International Jour-nal of Engineering Science, 87, 83-102 (2015)

[26] Dehrouyeh-Semnani, A. M., and Nikkhah-Bahrami, $M$. The influence of size-dependent shear deformation on mechanical behavior of microstructures-dependent beam based on modified couple stress theory. Composite Structures, 123, 325-336 (2015) 